\title{
Topographical Disorientation in Mild Cognitive Impairment: A Voxel-Based Morphometry Study
}

\author{
Tae-Sung Lim, MD; ; Giuseppe laria, $\mathrm{PhD}^{\mathrm{b}}$; So Young Moon, MD, $\mathrm{PhD}^{\mathrm{a}}$ \\ a'Department of Neurology, Ajou University School of Medicine, Suwon, Korea \\ ${ }^{\mathrm{b} D e p a r t m e n t}$ of Psychology, University of Calgary, Calgary, Canada
}

$\begin{array}{ll}\text { Received } & \text { April 6, 2010 } \\ \text { Revised } & \text { August 9, 2010 } \\ \text { Accepted } & \text { August 9, 2010 }\end{array}$

\section{Correspondence}

So Young Moon, $\mathrm{MD}, \mathrm{PhD}$

Department of Neurology,

Ajou University School of Medicine,

San 5 Woncheon-dong,

Yeongtong-gu, Suwon 443-721,

Korea

Tel +82-31-219-5175

Fax +82-31-219-5178

E-mail symoon.bv@gmail.com
Background and Purpose To assess the neural substrates underlying topographical disorientation (TD) in patients affected by mild cognitive impairment (MCI), forty-one patients diagnosed with MCI and 24 healthy control individuals were recruited.

Methods TD was assessed clinically in all participants. Neurological and neuropsychological evaluations and a volumetric-head magnetic resonance imaging scan were performed in each participant. Voxel-based morphometry was used to compare patterns of gray-matter atrophy between patients with and without TD, and a group of normal controls.

Results We found TD in 17 out of the $41 \mathrm{MCI}$ patients (41.4\%). The functional abilities were significantly impaired in MCI patients with TD compared to in MCI patients without TD. Voxel-based morphometry analyses showed that the presence of TD in MCI patients is associated with loss of gray matter in the medial temporal regions, including the hippocampus and parahippocampal cortex, the fusiform gyrus, the inferior occipital gyrus, the amygdala, and the cerebellum.

Conclusions The findings found in this study represent the first evidence that the presence of TD in patients with MCI is associated with loss of gray matter in those brain regions that have been documented to be responsible for orientation in both neuropsychological and neuroimaging studies.

$\mathrm{J}$ Clin Neurol 2010;6:204-211

Key Words mild cognitive impairment, topographical disorientation, voxel-based morphometry, dementia.

\section{Introduction}

Topographical disorientation (TD) refers to an individual's inability to orient in the environment. ${ }^{1}$ As reported in a current taxonomy, ${ }^{2} \mathrm{TD}$ may occur following lesions in different parts of the brain including medial temporal regions such as the hippocampus and parahippocampal cortex, the fusiform gyrus, and more-posterior temporo-occipital regions including the lingual gyrus, posterior cingulate gyrus, and posterior parietal cortex. Lesions in these selective brain regions may result in specific cognitive impairments such as anterograde disorientation, ${ }^{3}$ landmarks agnosia ${ }^{4}$ heading disorientation, ${ }^{5}$ or egocentric disorientation, ${ }^{6}$ which severely affect the individuals' ability to orient in familiar or unfamiliar surroundings. ${ }^{7}$

Similar cerebral regions causing TD in patients with acqu- ired brain damage have been shown to be involved in the neurodegenerative process typical of patients affected by mild cognitive impairment (MCI) progressing to Alzheimer's disease (AD) ${ }^{8-12}$ Due to this neuroanatomical similarity and evidence of MCI patients manifesting with orientation impairment, it has been suggested that TD occurs early in the development of $\mathrm{AD}$ and can be used for detecting the disease and monitoring its progression. ${ }^{13}$ Although this may be a reasonable speculation, there are no data on assessments of the neural substrates of TD in patients with MCI using an unbiased neuroimaging analysis methodology.

In the present study, we first clinically assessed the frequency of TD in a group of patients diagnosed with MCI. We then used voxel-based morphometry (VBM) to compare the brain morphology of MCI patients with (MCI-TD) and without (MCI- 
noTD) TD in order to verify the hypothesis that the presence of TD in MCI is associated with a loss of gray matter (GM) in those brain regions responsible for orientation.

\section{Methods}

\section{Participants}

We recruited 41 patients (mean age, 69 years) diagnosed with MCI between March 2007 and December 2008 (Ajou University Hospital, Suwon, Korea). MCI was diagnosed according to the following criteria proposed by Petersen: ${ }^{14}$

1) Subjective cognitive complaint by the patient or his/her caregiver.

2) Normal general cognitive function above the 16th percentile on the Korean version of the Mini-Mental State Examination (K-MMSE).

3) Normal activities of daily living (ADL) assessed both clinically and on a standardized scale (as described below).

4) Objective cognitive decline below the 16th percentile on neuropsychological tests.

5) Exclusion of dementia.

The absence of secondary causes of cognitive deficits was assessed by laboratory tests including complete blood count, blood chemistry, vitamin $\mathrm{B}_{12} /$ folate, syphilis serology, and thyroid function tests. Brain magnetic resonance imaging (MRI) scans were performed to confirm the absence of structural lesions such as territorial cerebral infarction, brain tumor, or vascular malformation.

The study participants comprised a group of 24 healthy control participants (mean age, 68 years) with no history of neurological or psychiatric illnesses and with no cognitive decline, as assessed by an extensive neuropsychological evaluation. No healthy control participants complained of TD.

Informed consent to participate in the study was obtained from all subjects, as approved by the Institutional Review Board of Ajou University Hospital.

\section{Procedure}

Participants underwent three sessions. During the first session, an experienced clinician (Moon SY) obtained a history of cognitive and behavioral functioning, and performed neurological examinations including ADL and the K-MMSE. ${ }^{15}$ The interview for ADL included additional items gathering information regarding patients' medications at the time of testing, and the patients' ability with respect to financial management, shopping, and use of mass transportation. In the second session, participants underwent a neuropsychological evaluation, blood tests, and MRI scans, including one volumetric-head MRI scan. In addition to the standard neuropsychological evaluation (described below), the neuropsychology team independently per- formed a clinical interview using semistructured questionnaires to assess cognitive, behavioral, and functional skills. During the same session, the neuropsychology team completed the Neuropsychiatric Inventory, ${ }^{16}$ the Clinical Dementia Rating Scale, ${ }^{17}$ and the ADL scale. The ADL scale adopted in this study consisted of the Seoul Instrumental ADL (S-IADL), which included 15 items rated from 0 (best) to 3 (worst; the final score of the ADL scale is obtained by summing the scores for each item). This scale has been standardized with a cutoff point of 7.5. ${ }^{18}$ Among the semistructured questionnaires, an interview was included to collect information about the patients' navigational skills from the patients themselves or from their primary caregivers (i.e., spouse or cohabitant). The presence of TD was determined by the question: "Does the patient have problems recognizing roads?" The term "recognizing roads" in Korean is a colloquial phrase meaning the ability to find one's way competently in an environment without hesitation in heading toward a destination or when returning home. We also asked them whether the patient experienced TD in an unfamiliar or familiar environment, or indoors. Although TD is commonly assessed by using objective measurements in virtual reality tests, previous studies have made use of a clinical approach similar to the one described here. ${ }^{19}$ This clinical approach is a reliable way of assessing the patients' ability to orient in their own surroundings, without exposing them to new tools or asking them to perform selective tests requiring different skills.

Finally, during the third session, participants returned to the clinic to receive the results of their neuropsychological assessments, blood tests, and MRI scans, which led to a consensus for the diagnosis of MCI.

\section{Neuropsychological evaluation}

All patients and controls underwent a standardized neuropsychological evaluation, the Seoul Neuropsychological Screening Battery. ${ }^{15}$ The battery, of which norms are based on 447 healthy controls, includes tests assessing attention, language, praxis, four elements of Gerstmann syndrome, visuoconstructive functions, verbal and visual memory, and frontal/executive function. Among these, the scorable tests included the digit span (forward and backward), the Korean version of the Boston Naming Test, ${ }^{20}$ the Rey-Osterrieth Complex Figure Test (copying, immediate and 20-min delayed recall, and recognition), the Seoul Verbal Learning Test (three learning-free recall trials of 12 words, 20-min delayed recall trial for these 12 items, and a recognition test), the phonemic and semantic Controlled Oral Word Association Test, and the Stroop Test (word and color reading of 112 items during a 2-min period). Patients' performances were considered impaired when they scored below the 16 th percentiles of healthy controls matched for age, gender, and education. 


\section{Behavioral statistical analysis}

Patients were classified as MCI-TD or MCI-noTD. We used the analysis of variance test to compare the means of groups with continuous measurements, and the chi-square test to compare groups performances for categorical measurements. Post hoc analysis was conducted using the Tukey test. The level of statistical significance was set at $p<0.05$. Analyses were performed using commercially available software [SPSS, version 12.0 (SPSS Inc, Chicago, IL, USA)].

\section{VBM: image acquisition and analyses}

MRI structural brain images were acquired using a 3.0-tesla Philips Achieva MRI scanner located at the Ajou University Hospital. Three-dimensional coronal high-resolution spoiled gradient echo sequences were acquired (excitation time, 4.59 $\mathrm{ms}$; relaxation time, $9.86 \mathrm{~ms}$; slice thickness, $1 \mathrm{~mm}$; 162 slices; flip angle, $8^{\circ}$; field of view, $224 \mathrm{~mm}$; and image matrix $224 \times$ 224 pixels, which covered the entire brain). An optimized VBM method was applied and implemented using SPM2 software (http://www.fil.ion.ucl.ac.uk/spm). ${ }^{21,22}$ Several preprocessing steps were performed to ready the data for statistical analysis, including spatial normalization and segmentation. The spatial normalization step transforms all images into the same stereotactic space by registering each of the images to the same specific template, and the segmentation of the brain into GM, white matter (WM), and cerebrospinal fluid (CSF) is performed using the voxel intensities combined with a priori knowledge of the spatial distribution of these tissues, derived from probability maps. In order to reduce any potential normalization or segmentation bias across the disease groups, customized templates and prior probability maps were created from all subjects in the study, including the controls and the semantic dementia patients. To create the customized template and prior probability maps, all images were registered to the Montreal Neurological Institute (MNI) template using a 12 degrees of freedom (df) affine transformation and segmented into GM, WM, and CSF using the MNI prior probability maps. GM images were normalized to the MNI GM template using a nonlinear discrete cosine transformation. The normalization parameters were applied to the original whole head and the images were resegmented. Average images were created of the whole head, GM, WM, and CSF, and smoothed using an 8-mm full-width-at-half-maximum smoothing kernel. The average whole-head image becomes the customized template, and the average GM, WM, and CSF images are then used as the customized prior probability maps for subsequent segmentations.

All images were then registered to the customized whole brain template using a 12-df affine transformation and segmented using the customized prior probability maps. The GM images were normalized to the custom GM template us- ing a nonlinear discrete cosine transformation. The normalization parameters were then applied to the original whole head and the images were resegmented. All of the GM images were modulated and smoothed with an 8-mm full-width-athalf-maximum smoothing kernel. In addition, a reinitialization routine was implemented using the parameters from the initial normalization to the MNI template (performed to generate the customized template) to initialize the normalization to the custom template. ${ }^{22}$ A single-subject condition and covariate model, including age, gender, and total intracranial volume as covariates, was used to compare the smoothed modulated GM images between the MCI-TD and controls ( $p<0.001$, uncorrected for multiple comparisons), and between the MCI-noTD and controls ( $p<0.001$, uncorrected for multiple comparisons). A direct comparison was also performed between MCI-TD and MCI-noTD $(p<0.01$, uncorrected for multiple comparisons).

\section{Results}

TD was found in 17 out of the 41 patients (41.4\%) affected by MCI. MCI-TD patients reported getting lost in large-scale unfamiliar $(82.4 \%)$ and familiar (29.4\%) surroundings, as well as in small-scale familiar environments such as a house (11.8\%). Patients referred to familiar surroundings as places that they have visited daily for several years, and unfamiliar surroundings were defined as places were patients had been several times sporadically over the past year. A more detailed interview revealed that patients reported getting lost when no remarkable landmarks were available in the environment, or when their navigation included several passages and turns, such as those required in a street market, a department store, or an apartment complex. Direct comparisons between MCI-TD and MCI-noTD patients revealed no significant difference with respect to age, gender, education, and general cognitive ability as measured by the K-MMSE. However, S-IADL scores revealed that the functional abilities were significantly impaired for MCI-TD patients relative to MCI-noTD patients. Table 1 gives the demographic data of the patients and controls, their K-MMSE and S-IADL scores, and the occurrence of TD in familiar and unfamiliar surroundings.

MCI-TD and MCI-noTD patients performed similarly in the neuropsychological tests. No significant differences were found in any of the tests available in the present neuropsychological battery. The performances of patients and controls in the tests included in the neuropsychological evaluation are reported in Table 2.

VBM revealed loss of GM bilaterally in the temporo-occipital regions when MCI-TD patients were compared to healthy controls $(p<0.001$, uncorrected for multiple compari- 
Table 1. Participants' demographic data, neurological evaluation scores, and prevalence of topographical disorientation

\begin{tabular}{|c|c|c|c|c|}
\hline & $\mathrm{MCl}-\mathrm{TD}(n=17)$ & MCl-noTD $(n=24)$ & Controls $(n=24)$ & $p$ \\
\hline Age (years) & $69.3 \pm 6.0$ & $68.7 \pm 7.4$ & $67.5 \pm 8.5$ & 0.781 \\
\hline Gender, female (\%) & $13(72.2)$ & $16(64.0)$ & $16(66.7)$ & 0.791 \\
\hline Education (years) & $8.6 \pm 5.2$ & $7.0 \pm 4.6$ & $7.5 \pm 5.2$ & 0.271 \\
\hline K-MMSE score & $25.8 \pm 1.9$ & $27.1 \pm 2.3$ & $29.1 \pm 1.8$ & $0.048^{t, \ddagger}$ \\
\hline S-IADL score & $5.7 \pm 1.1$ & $3.4 \pm 1.8$ & $1.5 \pm 2.1$ & $0.014^{*,+, \neq}$ \\
\hline TD-unfamiliar places [n (\%)] & $14(82.3)$ & - & - & - \\
\hline TD-familiar places [n (\%)] & $5(29.4)$ & - & - & - \\
\hline TD-home [n (\%)] & $2(11.7)$ & - & - & - \\
\hline
\end{tabular}

Data are mean \pm SD values except where stated otherwise.

*Significant difference between the two MCl groups, tSignificant difference between the MCl-TD and control groups, ₹Significant difference between the MCl-noTD and control groups.

TD: topographical disorientation, MCI-TD: mild cognitive impairment with TD, MCl-noTD: mild cognitive impairment without TD, K-MMSE: Korean version of the Mini-Mental State Examination, S-IADL: Seoul Instrumental Activities of Daily Living questionnaire.

Table 2. Neuropsychological assessment and participants' scores

\begin{tabular}{|c|c|c|c|c|}
\hline Neuropsychological test scores & MCl-TD $(n=19)$ & MCl-noTD $(n=20)$ & Controls $(n=20)$ & $p$ \\
\hline \multicolumn{5}{|l|}{ Attention } \\
\hline Digit-span (forward) & $5.5 \pm 1.5$ & $5.8 \pm 1.2$ & $6.3 \pm 1.4$ & $0.018^{*, \dagger}$ \\
\hline Digit-span (backward) & $2.5 \pm 1.2$ & $3.0 \pm 1.3$ & $3.4 \pm 0.9$ & $0.030^{*, t}$ \\
\hline \multicolumn{5}{|l|}{ Language and related functions } \\
\hline K-BNT (60) & $33.8 \pm 8.4$ & $37.1 \pm 11.8$ & $44.8 \pm 9.0$ & $0.021^{*, \dagger}$ \\
\hline \multicolumn{5}{|l|}{ Visuospatial functions } \\
\hline Copy of RCFT (36) & $26.0 \pm 10.7$ & $27.0 \pm 11.9$ & $31.1 \pm 6.4$ & $0.033^{*, \dagger}$ \\
\hline \multicolumn{5}{|l|}{ Memory functions } \\
\hline SVLT-IR $(12+12+12)$ & $14.1 \pm 3.9$ & $16.3 \pm 3.7$ & $20.3 \pm 4.3$ & $0.020^{*, \dagger}$ \\
\hline SVLT-DR (12) & $4.0 \pm 2.0$ & $3.8 \pm 2.3$ & $6.5 \pm 2.8$ & $0.028^{*, \dagger}$ \\
\hline Recognition (TP-FP) & $18.7 \pm 3.1$ & $19.4 \pm 3.0$ & $21.0 \pm 1.9$ & $0.039^{*, \dagger}$ \\
\hline RCFT-IR (36) & $8.2 \pm 8.2$ & $9.8 \pm 5.9$ & $15.4 \pm 6.9$ & $0.027^{*, \dagger}$ \\
\hline RCFT-DR (36) & $8.6 \pm 7.4$ & $9.7 \pm 6.3$ & $15.6 \pm 7.1$ & $0.017^{*, t}$ \\
\hline Recognition (TP-FP) & $18.1 \pm 2.2$ & $18.0 \pm 2.2$ & $19.9 \pm 2.0$ & $0.016^{*, t}$ \\
\hline \multicolumn{5}{|l|}{ Frontal functions } \\
\hline COWAT-A & $13.5 \pm 3.8$ & $12.3 \pm 4.0$ & $18.0 \pm 4.5$ & $0.023^{*, t}$ \\
\hline COWAT-S & $14.2 \pm 4.6$ & $13.6 \pm 4.6$ & $19.0 \pm 4.2$ & $0.011^{*, \dagger}$ \\
\hline Stroop Test C (112) & $70.5 \pm 17.6$ & $60.6 \pm 25.0$ & $90.5 \pm 17.0$ & $0.003^{*, t}$ \\
\hline
\end{tabular}

Performance of $\mathrm{MCl}$-TD patients, $\mathrm{MCl}$-noTD patients, and controls in the tests included in the neuropsychological evaluation. Data are mean $\pm S D$ values. Significant difference between the two $\mathrm{MCl}$ groups.

*Significant difference between the MCl-TD and control groups, ‡Significant difference between the MCI-noTD and control groups.

SVLT: Seoul Verbal Learning Test, K-BNT: Korean version of the Boston Naming Test, DR: delayed recall, IR: immediate recall, TP: true positive, FP: false positive, RCFT: Rey-Osterrieth Complex Figure Test, COWAT-A: Controlled Oral Word Association Test-phenomic, COWAT-B: Controlled Oral Word Association Test-semantic.

sons). In the right hemisphere, MCI-TD patients exhibited loss of GM from the anterior temporal pole, through the medial temporal regions (including the parahippocampal and fusiform gyri), extending to the middle and inferior temporal gyri toward the most posterior regions of the fusiform and inferior occipital gyri (Fig. 1). Similar regions were involved in the left hemisphere, although to a lesser extent. In addition, loss of GM was observed in the right amygdala, left angular gyrus, and cerebellum. On the other hand, MCI-noTD patients exhibited a loss of GM that was restricted to the left frontal and occipital regions when compared to the group of healthy con- trols ( $p<0.001$, uncorrected for multiple comparisons) (Fig. 2), which is consistent with previous VBM studies. ${ }^{23}$ The inverse contrast between MCI-TD or MCI-noTD patients and controls did not show any significant areas. As expected, direct comparisons between the two MCI groups (i.e., with and without TD) revealed that those with TD exhibited a greater level of GM loss bilaterally in the temporo-occipital regions ( $p<0.01$, uncorrected for multiple comparisons). These regions included the hippocampi and parahippocampal gyri, the fusiform gyrus, the inferior occipital gyrus, the amygdala, and the cerebellum (Fig. 3). The inverse comparison (MCI-noTD 

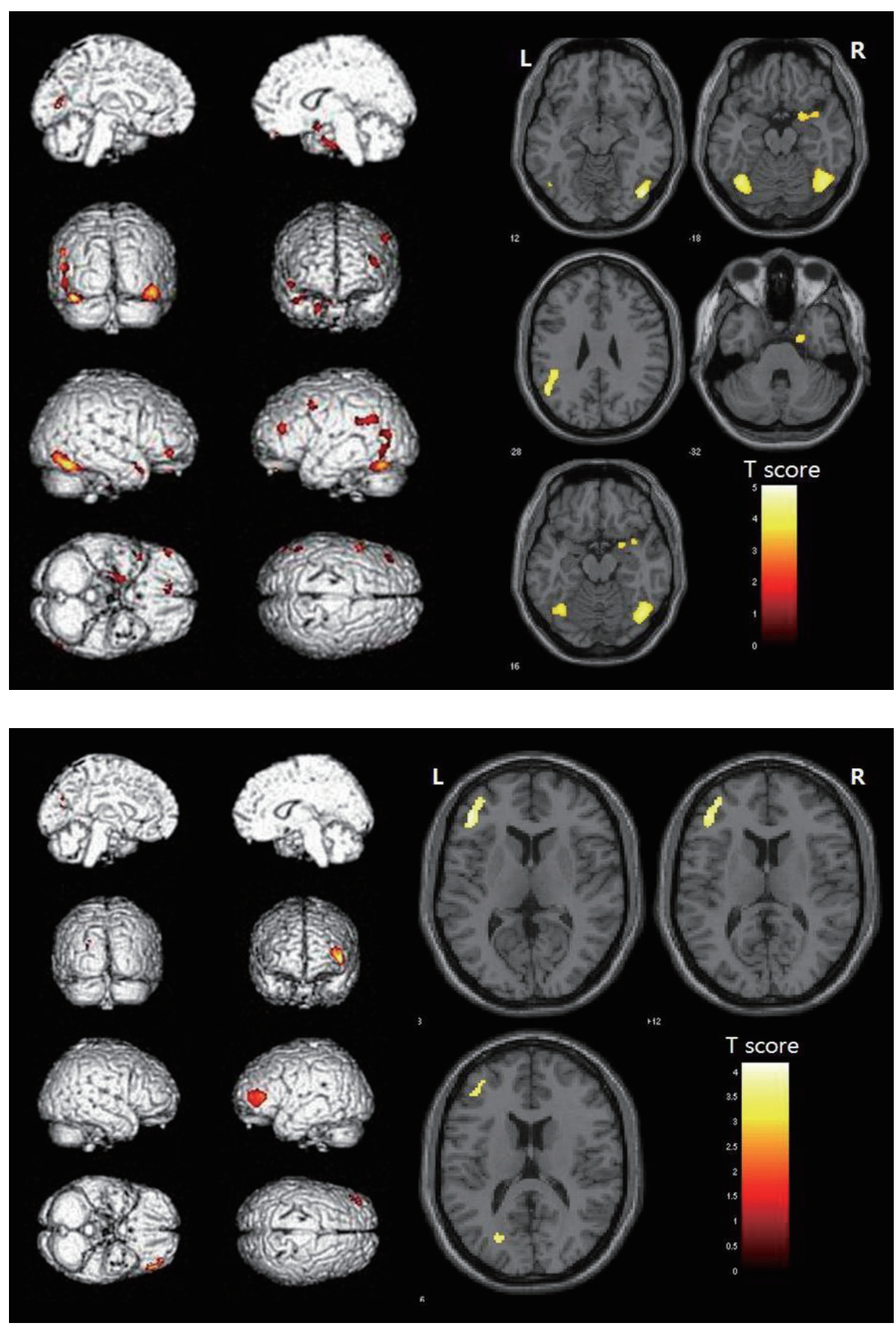

Fig. 1. Patterns of statistically significant loss of gray matter in patients with mild cognitive impairment (MCl) in combination with topographical disorientation (TD) compared to healthy control subjects $(p<0.001$, uncorrected for multiple comparisons). In the right hemisphere, $\mathrm{MCl}-\mathrm{TD}$ patients exhibited loss of gray matter from the anterior temporal pole, through the medial temporal regions (including the parahippocampal and fusiform gyri), extending to the middle and inferior temporal gyri toward the most posterior regions of the fusiform and inferior occipital gyri. Similar regions were involved in the left hemisphere, although to a lesser extent. In addition, gray matter loss was observed in the right amygdala, left angular gyrus, and cerebellum. versus MCI-TD) did not reveal any significant difference in GM loss.

\section{Discussion}

The aims of the present study were to determine the prevalence of TD in MCI patients and verify the hypothesis that TD is correlated with the loss of GM in brain regions reported to be responsible for orientation in humans. We found that $41.9 \%$ of patients diagnosed with MCI reported getting lost on a regular basis. VBM analyses revealed that the presence of TD in MCI patients is associated with loss of GM in the medial temporal regions, including the hippocampus and parahippocampal cortex, and the fusiform and inferior occipital gyri; additional loss of GM was observed in the cerebellum and the amygdala.

The morphological findings described herein are consistent with structural lesions causing TD in patients with acquired brain damage. Indeed, as described in a current taxono$\mathrm{my},{ }^{2}$ lesions to the hippocampus and parahippocampal cortex result in a selective topographical disorder known as antero- 
Fig. 3. Regions of gray matter loss in mild cognitive impairment-topographical disorientation(MCl-TD) patients compared to MCl-noTD patients ( $p<0.01$, uncorrected for multiple comparisons). $\mathrm{MCl}$ TD patients exhibited a greater loss of gray matter loss bilaterally in the temporo-occipital regions. These regions included the hippocampi and parahippocampal gyri, the fusiform gyrus, the inferior occipital gyrus, the amygdala, and the cerebellum.

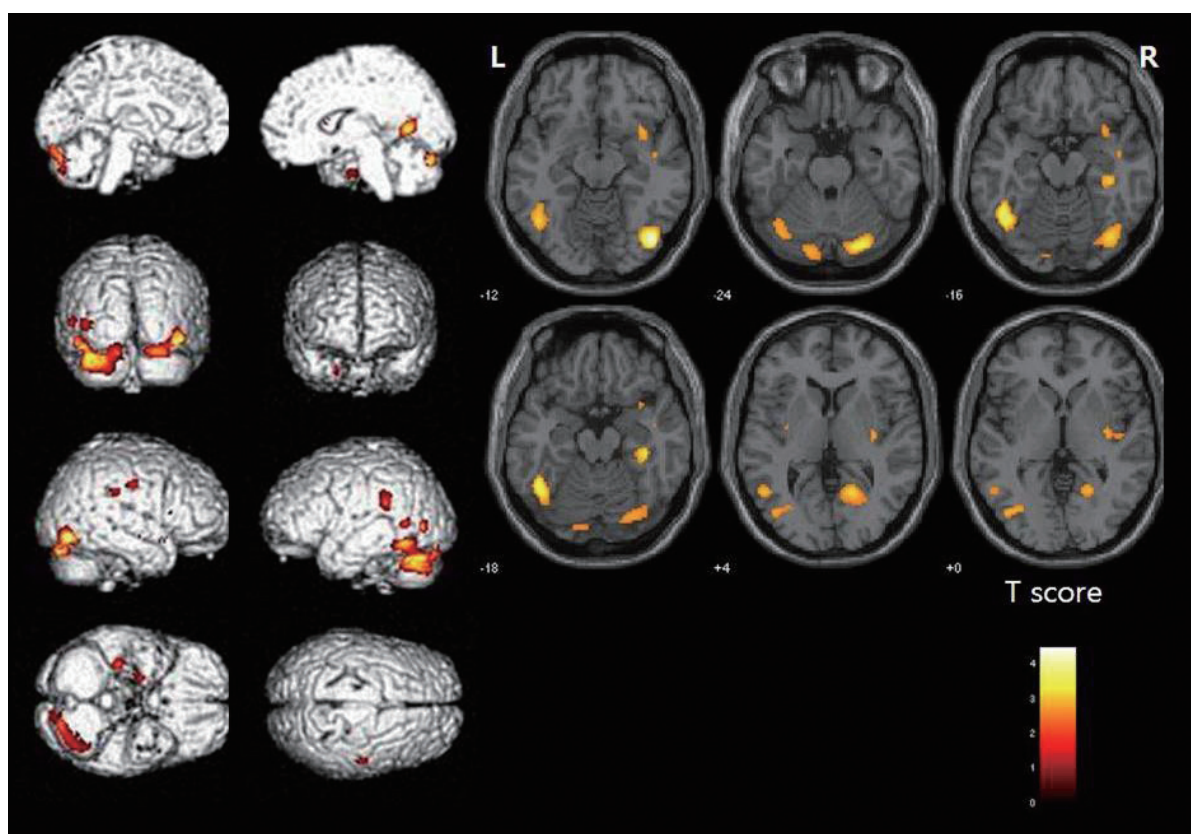

grade disorientation, which is the impaired ability to learn paths in a novel environment. ${ }^{3}$ Brain damage in the fusiform and inferior occipital gyri (including the lingual gyrus) impair the recognition of landmarks, resulting in landmark agnosia ${ }^{4}$ and adversely affecting the individual's ability to orient in both familiar and unfamiliar surroundings. Finally, data obtained from both human patients and animal models support that the cerebellum plays a critical role in spatial navigation, suggesting a specific involvement in the process of spatial learning. ${ }^{24-26}$ Our findings are also consistent with the most recent neuroimaging data describing the neural mechanisms involved in orientation and navigation in healthy individuals. ${ }^{27,28}$ Within an extensive neural network, the hippocampus and parahippocampal cortex have been shown to be critical for the acquisition and use of a mental representation of the environment (i.e., a cognitive map) $)^{29,30}$ and place recognition, ${ }^{31}$ respectively. Similarly, more-posterior regions including the fusiform and the lingual gyrus, ${ }^{32-35}$ as well as the cerebellum, ${ }^{25,36}$ have been found to play an important role in the processing and recognition of spatial information relevant for orientation. Consistent with these previous neuropsychological and neuroimaging data, to our knowledge the VBM findings reported herein provide the first evidence that the presence of TD in MCI patients is associated with loss of GM in those brain regions known to be critical for human orientation.

These findings may have relevant clinical implications for monitoring and predicting the MCI patient's progression to AD. Indeed, consistent with previous data, ${ }^{37-40}$ a recent study showed that the GM loss in a group of amnestic MCI patients who developed $\mathrm{AD}$ occurred primarily in medial temporal regions such as the hippocampal complex, the amygdala, and the fusiform gyrus. ${ }^{12}$ Early changes in the morphology of these brain structures can be detected 3 years before the diagnosis of $\mathrm{AD}$ and are suggested to be useful biomarkers for monitoring the progression of this disease. ${ }^{12}$ These selective brain regions markedly overlap with the loss of GM that we observed in our MCI-TD patients.

Our findings are also consistent with those of a recent study documenting the presence of TD in patients affected by amnestic MCI. ${ }^{13}$ Since there is an increased risk of amnestic MCI patients in developing AD (compared to nonamnestic MCIs), ${ }^{14}$ and there is evidence of orientation impairments occurring early in the development of $\mathrm{AD},{ }^{13}$ the authors suggest that assessment of TD in amnestic MCI is useful for monitoring the progression of the disease, and will eventually be used to detect $\mathrm{AD}$ at a presymptomatic stage. ${ }^{13}$ However, it should be noted that patients diagnosed with nonamnestic MCI may also develop $\mathrm{AD}$, although to a lesser extent compared to amnestic MCI patients. ${ }^{41}$ Our finding that TD is associated with loss of GM in those brain regions thought to be biomarkers of $\mathrm{AD}$, irrespective of the MCI subgroup, suggests that orientation impairments are also useful for monitoring the progression of the disease to $\mathrm{AD}$ in nonamnestic $\mathrm{MCI}$.

As a final note, the loss of GM in MCI-TD patients documented here involves brain regions that are not known to selectively subserve orientation skills, but are involved in other cognitive functions. Impaired performances could therefore have been expected when these patients performed other neuropsychological tests evaluating other functioning domains; this was not the case. We attribute this to the complexity of the phenomenon of orientation and navigational as compared to single and more selective cognitive functions. Indeed, ori- 
entation requires the cooperation of many cognitive skills during navigation, resulting in a complex neural network that is responsible for this complex behavior. The loss of GM in some brain regions that form part of that complex neural network may affect orientation skills without resulting in a severe and selective cognitive defect, which in turn may be observed in the case of brain damage resulting from vascular events.

We recognize that our study had some limitations. First, we adopted a subjective approach to define the existence of TD in our patients. In contrast to the objective assessment of TD, clinical interviews can be biased at the subjective sociocultural level. However, it has been reported that, with the exception of tests such as the Money Road Test ${ }^{42}$ and the Washington University Road test, ${ }^{43}$ most neuropsychological tests are insensitive to TD. ${ }^{44}$ Second, the average education level of our subjects was lower than those recruited into other studies. It is therefore difficult to generalize our findings to the overall population of patients with MCI. Finally, we performed SPM analyses without correcting for multiple comparisons, since we found no areas of significant difference when using the corrected criteria. Nonetheless, we considered it meaningful to provide the areas of significant difference even without the corrected criteria in patients with $\mathrm{MCI}$, since previous studies have shown that the areas associated with patients' complaints are known to be related to TD.

\section{Conflicts of Interest}

The authors have no financial conflicts of interest.

\section{REFERENCES}

1. Renzi ED. Disorders of space exploration and cognition. Chichester: Wiley, 1982.

2. Aguirre GK, D’Esposito M. Topographical disorientation: a synthesis and taxonomy. Brain 1999;122:1613-1628.

3. Habib M, Sirigu A. Pure topographical disorientation: a definition and anatomical basis. Cortex 1987;23:73-85.

4. Pallis CA. Impaired identification of faces and places with agnosia for colours; report of a case due to cerebral embolism. J Neurol Neurosurg Psychiatry 1955;18:218-224.

5. Takahashi N, Kawamura M, Shiota J, Kasahata N, Hirayama K. Pure topographic disorientation due to right retrosplenial lesion. Neurology 1997;49:464-469.

6. Stark M. Impairment of an Egocentric Map of Locations: Implications for Perception and Action. Cogn Neuropsychol 1996;13:481-524.

7. Barrash J. A historical review of topographical disorientation and its neuroanatomical correlates. J Clin Exp Neuropsychol 1998;20:807-827.

8. Huang C, Wahlund LO, Svensson L, Winblad B, Julin P. Cingulate cortex hypoperfusion predicts Alzheimer's disease in mild cognitive impairment. BMC Neurol 2002;2:9.

9. Trivedi MA, Wichmann AK, Torgerson BM, Ward MA, Schmitz TW, Ries ML, et al. Structural MRI discriminates individuals with Mild Cognitive Impairment from age-matched controls: a combined neuropsychological and voxel based morphometry study. Alzheimers Dement 2006;2:296-302.

10. Seo SW, Im K, Lee JM, Kim YH, Kim ST, Kim SY, et al. Cortical thickness in single- versus multiple-domain amnestic mild cognitive impair- ment. Neuroimage 2007;36:289-297.

11. Desikan RS, Fischl B, Cabral HJ, Kemper TL, Guttmann CR, Blacker $\mathrm{D}$, et al. MRI measures of temporoparietal regions show differential rates of atrophy during prodromal AD. Neurology 2008;71:819-825.

12. Whitwell JL, Przybelski SA, Weigand SD, Knopman DS, Boeve BF, Petersen RC, et al. 3D maps from multiple MRI illustrate changing atrophy patterns as subjects progress from mild cognitive impairment to Alzheimer's disease. Brain 2007;130:1777-1786.

13. Hort J, Laczó J, Vyhnálek M, Bojar M, Bures J, Vlcek K. Spatial navigation deficit in amnestic mild cognitive impairment. Proc Natl Acad Sci U S A 2007;104:4042-4047.

14. Petersen RC. Mild cognitive impairment as a diagnostic entity. J Intern Med 2004;256:183-194.

15. Kang Y, Na D. Seoul Neuropsychological Screening Battery. Incheon: Human Brain Research and Consulting, 2003.

16. Cummings JL, Mega M, Gray K, Rosenberg-Thompson S, Carusi DA, Gornbein J. The Neuropsychiatric Inventory: comprehensive assessment of psychopathology in dementia. Neurology 1994;44:2308-2314.

17. Morris JC. The Clinical Dementia Rating (CDR): current version and scoring rules. Neurology 1993;43:2412-2414.

18. Ku HM, Kim JH, Kwon EJ, Kim SH, Lee HS, Ko HJ, et al. A study on the reliability and validity of seoul-instrumental activities of daily living (S-IADL). J Korean Neuropsychiatr Assoc 2004;43:189-199.

19. Pai MC, Jacobs WJ. Topographical disorientation in community-residing patients with Alzheimer's disease. Int J Geriatr Psychiatry 2004; 19:250-255.

20. Kim H, Na D. Normative data on the Korean version of the Boston Naming Test. J Clin Exp Neuropsychol 1999;21:127-133.

21. Ashburner J, Friston KJ. Voxel-based morphometry--the methods. Neuroimage 2000;11:805-821.

22. Senjem ML, Gunter JL, Shiung MM, Petersen RC, Jack CR Jr. Comparison of different methodological implementations of voxel-based morphometry in neurodegenerative disease. Neuroimage 2005;26:600608 .

23. Bozzali M, Filippi M, Magnani G, Cercignani M, Franceschi M, Schiatti $\mathrm{E}$, et al. The contribution of voxel-based morphometry in staging patients with mild cognitive impairment. Neurology 2006;67:453-460.

24. Rondi-Reig L, Burguière E. Is the cerebellum ready for navigation? Prog Brain Res 2005;148:199-212.

25. Lee TM, Liu HL, Hung KN, Pu J, Ng YB, Mak AK, et al. The cerebellum's involvement in the judgment of spatial orientation: a functional magnetic resonance imaging study. Neuropsychologia 2005;43:18701877.

26. Burguière E, Arleo A, Hojjati M, Elgersma Y, De Zeeuw CI, Berthoz A, et al. Spatial navigation impairment in mice lacking cerebellar LTD: a motor adaptation deficit? Nat Neurosci 2005;8:1292-1294.

27. Aguirre GK, Zarahn E, D'Esposito M. An area within human ventral cortex sensitive to "building" stimuli: evidence and implications. $\mathrm{Neu}$ ron 1998;21:373-383.

28. Maguire EA. Hippocampal involvement in human topographical memory: evidence from functional imaging. Philos Trans R Soc Lond B Biol Sci 1997;352:1475-1480.

29. Iaria G, Chen JK, Guariglia C, Ptito A, Petrides M. Retrosplenial and hippocampal brain regions in human navigation: complementary functional contributions to the formation and use of cognitive maps. Eur $J$ Neurosci 2007;25:890-899.

30. Iaria G, Lanyon LJ, Fox CJ, Giaschi D, Barton JJ. Navigational skills correlate with hippocampal fractional anisotropy in humans. Hippocampus 2008;18:335-339.

31. Epstein RA, Parker WE, Feiler AM. Where am I now? Distinct roles for parahippocampal and retrosplenial cortices in place recognition. $J$ Neurosci 2007;27:6141-6149.

32. Ekstrom AD, Kahana MJ, Caplan JB, Fields TA, Isham EA, Newman $\mathrm{EL}$, et al. Cellular networks underlying human spatial navigation. $\mathrm{Na}$ ture 2003;425:184-188. 
33. Epstein R, Kanwisher N. A cortical representation of the local visual environment. Nature 1998;392:598-601.

34. Grön G, Wunderlich AP, Spitzer M, Tomczak R, Riepe MW. Brain activation during human navigation: gender-different neural networks as substrate of performance. Nat Neurosci 2000;3:404-408.

35. Zeki S. Cerebral akinetopsia (visual motion blindness). A review. Brain 1991;114:811-824.

36. Jahn K, Deutschländer A, Stephan T, Strupp M, Wiesmann M, Brandt T. Brain activation patterns during imagined stance and locomotion in functional magnetic resonance imaging. Neuroimage 2004;22:17221731.

37. Chételat G, Desgranges B, De La Sayette V, Viader F, Eustache F, Baron JC. Mapping gray matter loss with voxel-based morphometry in mild cognitive impairment. Neuroreport 2002;13:1939-1943.

38. Karas GB, Scheltens P, Rombouts SA, Visser PJ, van Schijndel RA, Fox NC, et al. Global and local gray matter loss in mild cognitive impairment and Alzheimer's disease. Neuroimage 2004;23:708-716.

39. Pennanen C, Testa C, Laakso MP, Hallikainen M, Helkala EL, Hän- ninen T, et al. A voxel based morphometry study on mild cognitive impairment. J Neurol Neurosurg Psychiatry 2005;76:11-14.

40. Bell-McGinty S, Lopez OL, Meltzer CC, Scanlon JM, Whyte EM, Dekosky ST, et al. Differential cortical atrophy in subgroups of mild cognitive impairment. Arch Neurol 2005;62:1393-1397.

41. Fischer P, Jungwirth S, Zehetmayer S, Weissgram S, Hoenigschnabl S, Gelpi E, et al. Conversion from subtypes of mild cognitive impairment to Alzheimer dementia. Neurology 2007;68:288-291.

42. Money J, Alexander D, Walker H. Astandardized road-map test of direction sense. Baltimore, MD: Johns Hopkins University Press, 1976.

43. Hunt LA, Murphy CF, Carr D, Ducherk JM, Buckles V, Morris JC. Environmental cueing may effect performance on a road test for drivers with dementia of the Alzheimer type. Alzheimer Dis Assoc Disord 1997;11 Suppl 1:13-16.

44. Nadolne MJ, Stringer AY. Ecologic validity in neuropsychological assessment: prediction of wayfinding. J Int Neuropsychol Soc 2001; 7: $675-682$. 